\title{
Voltage-Dependent Anion Channels Influence Cytotoxicity of ME-344, a Therapeutic Isoflavone ${ }^{[\mathrm{S}}$
}

\author{
Leilei Zhang, Danyelle M. Townsend, Morgan Morris, Eduardo N. Maldonado, Yu-Lin Jiang, \\ Ann-Marie Broome, Jennifer R. Bethard, Lauren E. Ball, and Kenneth D. Tew \\ Department of Cell and Molecular Pharmacology and Experimental Therapeutics (L.Z., M.M., E.N.M., Y.-L.J., A.-M.B., J.R.B., \\ L.E.B., K.D.T.) and Department of Pharmaceutical and Biomedical Sciences (D.M.T.), Medical University of South Carolina, \\ Charleston, South Carolina
}

Received March 17, 2020; accepted May 19, 2020

\begin{abstract}
ME-344 is a second-generation cytotoxic isoflavone with anticancer activity promulgated through interference with mitochondrial functions. Using a click chemistry version of the drug together with affinity-enriched mass spectrometry, voltagedependent anion channels (VDACs) 1 and 2 were identified as drug targets. To determine the importance of VDAC1 or 2 to cytotoxicity, we used lung cancer cells that were either sensitive $(\mathrm{H} 460)$ or intrinsically resistant $(\mathrm{H} 596)$ to the drug. In $\mathrm{H} 460$ cells, depletion of VDAC1 and VDAC2 by small interfering RNA impacted ME-344 effects by diminishing generation of reactive oxygen species (ROS), preventing mitochondrial membrane potential dissipation, and moderating ME-344-induced cytotoxicity and mitochondrial-mediated
\end{abstract}

apoptosis. Mechanistically, VDAC1 and VDAC2 knockdown prevented ME-344-induced apoptosis by inhibiting Bax mitochondrial translocation and cytochrome $c$ release as well as apoptosis in these $\mathrm{H} 460$ cells. We conclude that VDAC1 and 2, as mediators of the response to oxidative stress, have roles in modulating ROS generation, Bax translocation, and cytochrome $c$ release during mitochondrial-mediated apoptosis caused by ME-344.

\section{SIGNIFICANCE STATEMENT}

Dissecting preclinical drug mechanisms are of significance in development of a drug toward eventual Food and Drug Administration approval.

\section{Introduction}

ME-344 as a synthetic isoflavone analog of phenoxodiol with structural similarities to genestein, is currently in clinical trial in cancer patients (Bendell et al., 2015; Diamond et al., 2017). One requirement toward attaining regulatory approval for the drug is to define its mechanism of action. Presently, it is apparent that mitochondria are directly impacted by drug treatment, but more than one molecular target has been identified. Possible interactive promiscuity is exemplified by reports that the drug induced inhibition of respiratory complexes (Alvero et al., 2011; Lim et al., 2015; Manevich et al., 2016; Navarro et al., 2016) and translocation of heme oxygenase 1 (HO-1) from rough endoplasmic reticulum to mitochondria (Manevich et al., 2016). In addition, ME-344 also causes

This work was supported by grants from National Institutes of Health National Institute of General Medical Sciences [Grant 5P20-GM103542-09, (COBRE in Oxidants, Redox Balance and Stress Signaling)], and support from the South Carolina Centers of Excellence program and was conducted in a facility constructed with the support from National Institutes of Health National Center for Research Resources Extramural Research Facilities Program [Grant C06-RR015455].

https://doi.org/10.1124/jpet.120.000009.

S This article has supplemental material available at jpet.aspetjournals.org. increased mitochondrial reactive oxygen species (ROS) production, Bax-mediated loss of mitochondrial membrane potential $\left(\Delta \Psi_{\mathrm{m}}\right)$ (Alvero et al., 2011; Zhang et al., 2019c), and inhibition of mitochondrial permeability transition (MPT) (Alvero et al., 2009; Zhang et al., 2019c). MPT is mediated through the opening/closing of a supramolecular complex permeability transition pore (PTP) between the inner and outer mitochondrial membranes. The molecular entities of the MPT pore are still debated, although hexokinase, voltagedependent anion channels (VDACs), adenine nucleotide translocase, and cyclophilin D have been proposed to be components of the pore (Zoratti et al., 2005; Brenner and Grimm, 2006). Bax, as a member of the Bcl-2 protein family, exists in equilibrium between mitochondria and cytosol (Schellenberg et al., 2013) and is also critical in maintaining MPT (Youle and Strasser, 2008).

We recently used a click chemistry version of ME-344 and showed that it binds to and inhibits HO-1. Proteomic analyses also indicated that VDAC1 and 2 were binding targets (Zhang et al., 2019b)(Zhang, 2019). VDAC, a highly conserved $\sim 30$-kDa protein with three isoforms in humans (VDAC1, 2, and 3), is the most abundant protein in the mitochondrial outer membrane. Respiratory substrates, such as cytosolic

ABBREVIATIONS: $\Delta \psi_{\mathrm{m}}$, mitochondrial membrane potential; ER, endoplasmic reticulum; FITC, fluorescein isothiocyanate; HO-1, heme oxygenase 1; JC-1, 5,5',6,6'-tetrachloro-1,1',3,3'-tetra-ethylbenzimidazolylcarbocyanine iodide; MPT, mitochondrial permeability transition; mPTP, mitochondrial PTP; PCR, polymerase chain reaction; PI, phosphatidylinositol; PTP, permeability transition pore; qPCR, quantitative PCR; ROS, reactive oxygen species; si, small interfering; TMRM, tetramethylrhodamine methylester; VDAC, voltage-dependent anion channel. 
ATP, ADP, and Pi entering mitochondria and mitochondrial ATP moving to the cytosol, cross the mitochondrial outer membrane through VDAC (Colombini, 1989). The distribution of VDAC isoforms varies from cell to cell and from cancer cell line to cancer cell line, but, in general, VDAC1 and VDAC2 are the most abundant isoforms. VDAC1, the most studied isoform, has been proposed to play important roles in several physiologic processes, including calcium signaling, regulation of cellular bioenergetics, and ROS control. VDAC2, implicated in the regulation of apoptosis, is also a major contributor of mitochondrial metabolism, whereas the minor isoform VDAC3 only recently has been shown to be a pore-forming channel (De Pinto et al., 2010; McCommis and Baines, 2012; Maldonado et al., 2013; Okazaki et al., 2015; Reina et al., 2016). Accumulating evidence indicates that VDACs play a role in determining cell survival (Rostovtseva and Colombini, 1996; Hodge and Colombini, 1997; Gincel et al., 2001) or apoptosis (Baek et al., 1997; Ding et al., 2001; Bae et al., 2003; Godbole et al., 2003; Zaid et al., 2005). Perhaps related to their interaction with the Bcl-2 family member Bax, VDAC1 and 2 have been implicated in regulation of apoptosis (Ma et al., 2014; Caterino et al., 2017). Silencing of VDAC1 or VDAC2 effectively prevents the association of Bcl-2 protein with mitochondria and inhibits Bax function and apoptosis induced by anticancer drugs (Tajeddine et al., 2008; Yuan et al., 2008; Chin et al., 2018; Dadsena et al., 2019a,b).

It has been recently proposed that VDAC operates as a switch for global control of mitochondrial metabolism in cancer cells. Moreover, small molecules that cause VDAC opening increase mitochondrial metabolism and generation of ROS and decrease levels of enhanced glycolysis, acting as antiWarburg compounds (Maldonado, 2017)(DeHart, 2017)(Fang, 2018)(Heslop, 2020)(Maldonado, 2017; DeHart et al., 2018; Maldonado and Fang, 2018; Heslop et al., 2020).

Since our previous click chemistry/proteomics analysis showed that ME-344 binds to VDAC1 and VDAC2, we undertook the present studies, focusing on these two isoforms to determine their relevance in drug-response patterns in lung cancer cell lines that are either intrinsically sensitive or resistant to ME-344.

\section{Materials and Methods}

Cell Lines. H460 large-cell lung cancer cells were from the National Cancer Institute (Frederick, MD), and H596 adenosquamous lung carcinoma cells were from American Type Culture Collection. Each collection of cells was maintained in RPMI1640 medium (Corning, NY) supplemented with $10 \%$ (v/v) FBS (Atlas), $100 \mathrm{U} / \mathrm{ml}$ penicillin, $100 \mathrm{mg} / \mathrm{ml}$ streptomycin, and $2 \mathrm{mM}$ L-glutamine. Cell lines were authenticated as described previously (Zhang et al., 2019b).

Antibodies. The following antibodies were used for immunoblots: Mouse monoclonal anti-ATP5A (ab110273) was from Abcam, Cambridge, MA; Mouse monoclonal anti-cytochrome c was from R\&D SYSTEMS, Minneapolis, MN; Mouse monoclonal anti-Bax (B9054) and mouse monoclonal anti- $\beta$-actin were from Sigma, St. Louis, MO; and IRDye $800 \mathrm{CW}$ Goat anti-Mouse IgG, IRDye $800 \mathrm{CW}$ Goat antiRabbit IgG, IRDye 680RD Goat anti-mouse IgG, and IRDye 680RD Goat anti-Rabbit IgG were all from LI-COR, Lincoln, NE.

Trypan Blue Assay. To determine the cell viability by vital dye exclusion tests, $20 \mu \mathrm{l}$ of cell suspension was mixed with $20 \mu \mathrm{l}$ of $0.4 \%$ trypan blue solution (Sigma-Aldrich) for 5 minutes. The number of viable cells was counted using a hemocytometer. Each experiment was carried out a minimum of three independent times. Mean and S.D. were calculated for each subculture.
ROS Detection. ROS detection was performed as previously described (Zhang et al., 2019b). Cells were treated with $1 \mathrm{mM} \mathrm{2} 2^{\prime}, 7^{\prime}$ dichlorodihydrofluorescein diacetate $\left(\mathrm{CM}-\mathrm{H}_{2}\right.$ DCFDA; Thermo Fisher Scientific) for 45 minutes, and this was followed by washing two times with PBS. The reduced CM- $\mathrm{H}_{2}$ DCFDA could be oxidized and converted into fluorescent $2^{\prime}, 7^{\prime}$-dichlorofluorescein by intracellular ROS. The fluorescent signals were detected by flow cytometer (Beckman Coulter). In total, 10,000 cells were analyzed per sample.

Small Interfering RNA Treatment. Cells grown to $70 \%$ $80 \%$ confluence were transfected with small interfering (si) RNA (50 nM) targeting VDAC1, VDAC2, and VDAC3 or control nontarget siRNA (Ambion, Austin, TX). Nontarget siRNA was Silencer Select Negative Control \#1 siRNA (Catalog 4390844). siRNAs for VDAC1, VDAC2, and VDAC3 were Silencer Select siRNAs: VDAC1, Catalog 4390824, ID: s14769; VDAC2, Catalog 4392420, ID: s14771; and VDAC3, Catalog 4392420, ID: s230730. siRNAs were reverse transfected using LipofectAMINE RNAiMAX Transfection Reagent according to the manufacturer's instructions (Invitrogen, Carlsbad, CA).

RNA Isolation and Quantitative Real-Time Reverse-Transcription Polymerase Chain Reaction. RNA isolation and real-time polymerase chain reaction (PCR) were performed as described previously (Zhang et al., 2019b). Total RNA was prepared using the Isolate II RNA Mini Kit (Bioline USA, Inc.), and cDNA was then synthesized with the iScript cDNA Synthesis Kit (Bio-Rad). Subsequently, quantification of gene expression was performed in duplicate using iQ SYBR Green Supermix (Bio-Rad) with detection on a MyiQ Real-Time PCR System (Bio-Rad). The reaction cycles used were $95^{\circ} \mathrm{C}$ for 5 minutes, 40 cycles at $95^{\circ} \mathrm{C}$ for 15 seconds, and $58^{\circ} \mathrm{C}$ for 1 minute, and this was followed by melt curve analysis. Relative gene expression quantification was based on the comparative threshold cycle method $(\triangle \triangle \mathrm{Ct})$ with normalization of the raw data to the housekeeping gene (18S ribosomal RNA).

Loading of Tetramethylrhodamine Methylester and Laser-Scanning Confocal Microscopy. H460 (drug-sensitive) and H596 (drug-resistant) cells plated in MatTek dishes were loaded with $200 \mathrm{nM}$ tetramethylrhodamine methylester (TMRM) for 30 minutes in modified Hank's balanced salt solution containing (in millimolars): $137 \mathrm{NaCl}, 0.35 \mathrm{Na}_{2} \mathrm{HPO}_{4}, 5.4 \mathrm{KCl}, 1 \mathrm{KH}_{2} \mathrm{PO}_{4}, 0.81$ $\mathrm{MgSO}_{4}, 0.95 \mathrm{CaCl}_{2}, 5.5$ glucose, $25 \mathrm{NaHCO}_{3}$, and HEPES 20, $\mathrm{pH} 7.4$, or complete growth media. After the initial loading, cells were maintained in $50 \mathrm{nM}$ TMRM to maintain the equilibrium of distribution (Lemasters, 2007)(Lemasters and Ramshesh, 2007). Cells incubated in modified Hank's balanced salt solution in a humidified $5 \% \mathrm{CO}_{2}$ /air at $37^{\circ} \mathrm{C}$ were imaged with a Zeiss LSM 880 NLO inverted laser-scanning confocal microscope (Thornwood, $\mathrm{NY)}$ with a $63 \times 1.4$ numerical aperture plan apochromat oil immersion lens. TMRM was excited at $561 \mathrm{~nm}$, and emission signal was detected with a Quasar multichannel spectral detector at $590-610 \mathrm{~nm}$ through a one-Airy unit diameter pinhole (Maldonado et al., 2013).

$5,5^{\prime}, 6,6^{\prime}$ 'Tetrachloro-1,1',3,3' -tetra-ethylbenzimidazolylcarbocyanine iodide Staining. Cells were stained with the mitochondrial membrane potential sensitive probe, JC-1 (5, 5' $, 6,6^{\prime}$-tetrachloro-1, $1^{\prime}, 3,3^{\prime}$ tetra-ethylbenzimidazolylcarbocyanine iodide) (Invitrogen), at a final concentration of $2 \mu \mathrm{M}$ in PBS for 15 minutes at $37^{\circ} \mathrm{C}$. After two PBS washes $(300 \mathrm{~g}, 5$ minutes), labeled cells were observed with an Olympus FV10i laser-scanning confocal. The aggregate red form of JC-1 has absorption/emission maxima of 585/590 $\mathrm{nm}$, whereas the green monomeric form is $510 / 527 \mathrm{~nm}$. Images acquired from confocal microscopy were measured and calculated for mean fluorescence intensities using ImageJ software version 1.41 (Rasband, W.S., ImageJ, U. S. National Institutes of Health, Bethesda, MD). For flow cytometry, JC-1 aggregates were measured in the fluorescence 2 channel, and JC-1 monomer was measured in the fluorescence 1 channel. Fluorescenceactivated cell sorter data were analyzed by CytExpert.

Apoptosis Assay. Annexin V and PI staining were performed according to the manufacturer's instructions (556547; BD Pharmingen). 
Cells were washed twice with cold PBS and then resuspended in $1 \times$ Binding Buffer at a concentration of $1 \times 10^{6}$ cells $/ \mathrm{ml}$. One hundred microliters of the solution $\left(1 \times 10^{5}\right.$ cells $)$ was transferred to a 5 -ml culture tube and $5 \mu \mathrm{l}$ of FITC Annexin V and $5 \mu \mathrm{l}$ PI were added. Cells were vortexed and incubated for 15 minutes at $25^{\circ} \mathrm{C}$ in the dark. Four hundred microliters of $1 \times$ Binding Buffer was added to each tube and subject to flow cytometry within 1 hour.

Purification of Mitochondria and Cytosol. Mitochondria and cytosol fractions were isolated as previously described (Wettmarshausen and Perocchi, 2017). Purities of cell fractions were determined by immunoblotting against $\beta$-actin (cytosol marker), ATP5A (mitochondrial marker), and sarco/endoplasmic reticulum (ER) $\mathrm{Ca}^{2+}$-ATPase (ER marker).

Immunoblotting. Immunoblotting was performed as previously described (Zhang et al., 2019b). Total soluble protein was quantitated by bicinchoninic acid protein assay (Pierce). Mitochondria and cytosol fractions were resolved in an SDS-loading buffer [ $80 \mathrm{mM}$ Tris-HCl, $\mathrm{pH}$ 6.8; $2 \%$ SDS; $10 \%$ glycerol; $0.02 \%$ bromophenol blue; $5 \mathrm{mM}$ Tris(2carboxyethyl)phosphine] and heated at $95^{\circ} \mathrm{C}$ for 5 minutes. Equal amounts $(50 \mu \mathrm{g})$ of protein were electrophoretically separated by SDSPAGE (Bio-Rad) and transferred onto low fluorescent polyvinylidene fluoride membranes (Millipore) by the Trans-Blot Turbo Transfer System (Bio-Rad). PVDF or nitrocellulose membranes were incubated in the Odyssey blocking buffer (LI-COR) for 1 hour to reduce nonspecific binding and then probed with appropriate primary antibodies at $4^{\circ} \mathrm{C}$ overnight. Immunoblots were then developed with infrared fluorescence IRDye secondary antibodies (LI-COR) at a dilution of 1 : 15,000 , imaged with a 2 -channel (red and green) infrared fluorescent Odyssey CLx imaging system (LI-COR), and quantified with ImageJ software (FIJI).

Click Chemistry and Affinity Enrichment Mass Spectrometry. Click chemistry and liquid chromatography tandem mass spectrometry were performed as previously described (Zhang et al., 2019b).
However, whereas the previous analysis focused only on drug-enriched proteins of $25-50 \mathrm{kDa}$, the present study was expanded. All proteins enriched by ME-344 beads (or negative controls) were fractionated into six gel bands before in-gel digestion and analyzed by liquid chromatography tandem mass spectrometry. Quantitative results were extracted and analyzed using MaxQuant and Perseus. Proteins were filtered to retain only those quantified in duplicate ME-344 enrichments, and missing values in the control enrichment were recorded as previously described (Zhang et al., 2019b).

Protein interaction and annotation networks were generated using a web-based tool, NDEx (Pratt et al., 2015, 2017; Pillich et al., 2017). Protein interactions associated with enriched proteins were extracted from multiple protein interaction data bases accessible in NDEx (BIOGRID, String, HumanNet, HIPPIE) and merged and visualized with Cytoscape 3.8.0 (Shannon et al., 2003; Otasek et al., 2019). Annotated gene-pathway relationships of VDAC1 and VDAC2 curated in the Comparative Toxicogenomics Database (Davis et al., 2019) were accessed through NDEx Integrated Query and visualized with Cytoscape.

Statistical Analysis. All measurements were collected from three independent experiments. Statistical analysis was performed using GraphPad Prism 6.0 and Microsoft Excel. Significant differences were determined using two-tailed $t$ tests. The results are expressed as the mean \pm S.D. Significance levels were established at a level of $P<0.05$.

\section{Results}

ME-344 Binds Directly to VDAC1 and 2. Using a monopropargylated derivative of ME-344 (M2F) (Supplemental Fig. 1) with azide agarose resin (Zhang et al., 2019b), we identified protein binding via affinity enrichment mass spectrometry. Our previous study focused on a single, differentially stained gel
A
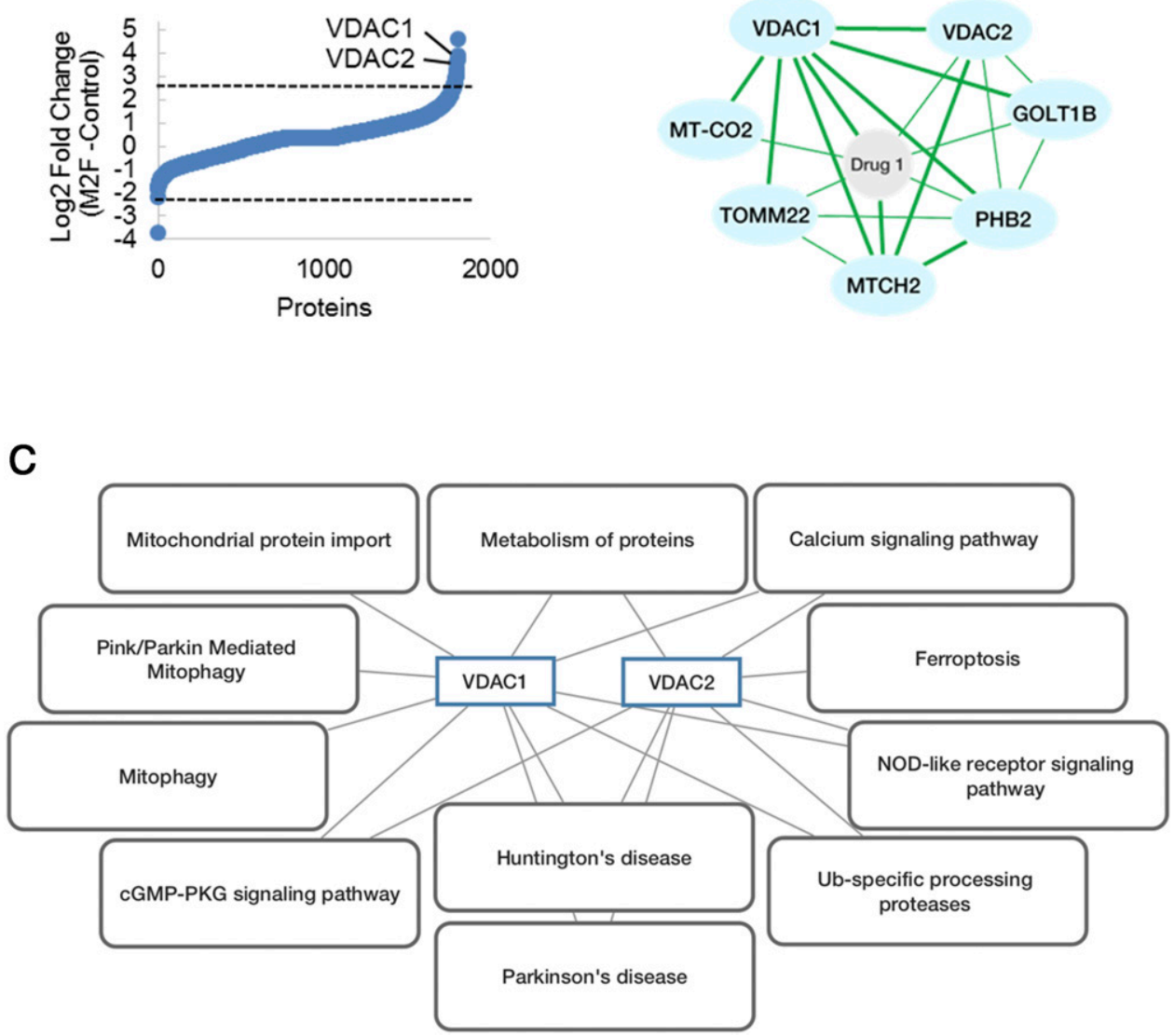

Fig. 1. VDAC1 and 2 were identified as ME-344 targets in H460 cells by using click chemistry and mass spectrometry. Affinity-enriched and gel-fractionated proteins between 10 and $250 \mathrm{kDa}$ were analyzed by LC/MS-MS and quantified by label-free proteomics. (A) Proteins with a $\log _{2}$ fold change in intensity $>2.5$ were considered enriched with M2F, as compared with control beads. (B) Twelve proteins enriched with M2F were queried for known protein interactions revealing VDAC 1 and 2 interactions with five of the other enriched proteins. This network of interactions was visualized with Cytoscape. (C) Functional annotations for VDAC1 and 2 extracted from the Reactome and Kyoto Encyclopedia of Genes and Genomes data bases. cGMP-PKG, Cyclic guanosine monophosphate protein kinase G;GOLT1B, Vesicle transport protein GOT1B;MTCH2, Mitochondrial carrier homolog 2;MT-CO2, Cytochrome c oxidase subunit 2;NOD, Nucleotide-binding and oligomerization domain;PHB2, Prohibitin2;TOMM22, Mitochondrial import receptor subunit TOM22 homolog. 
A

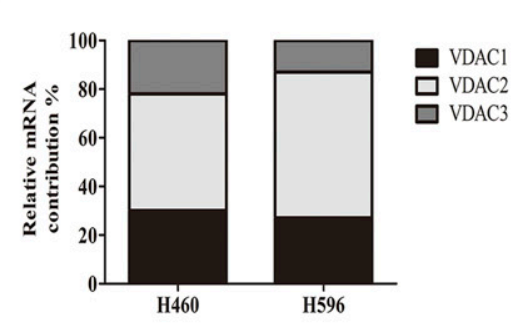

D

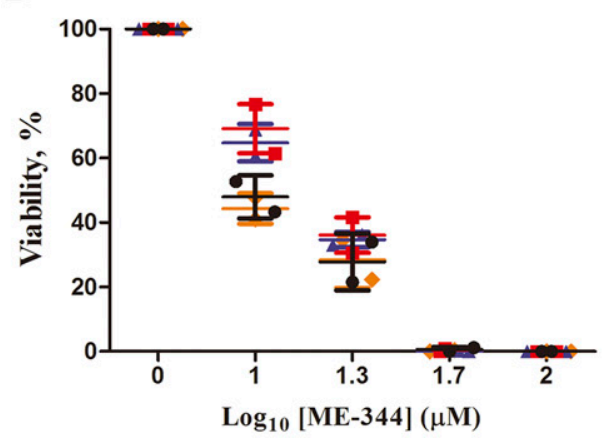

F

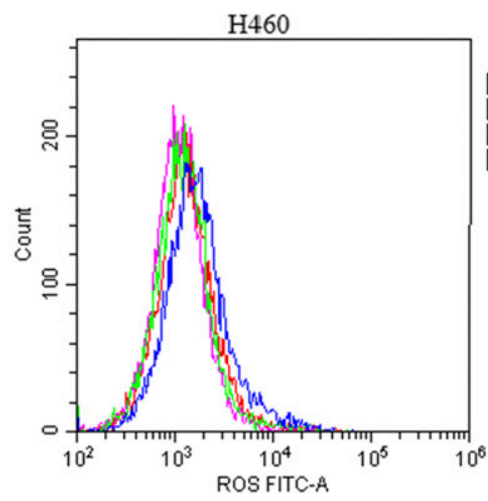

H

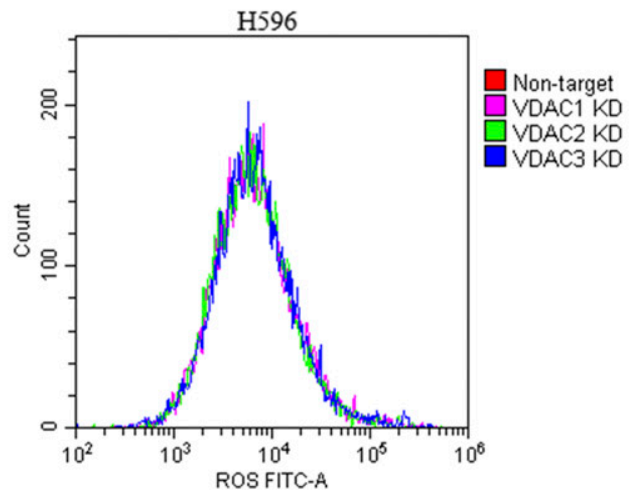

B
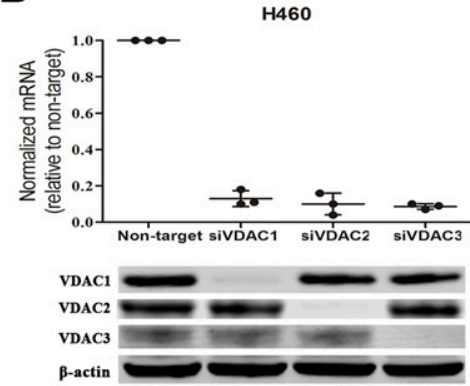

C
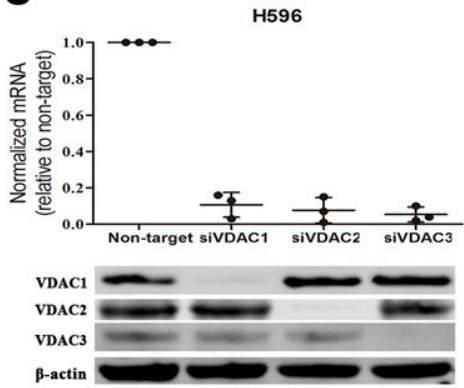

H596

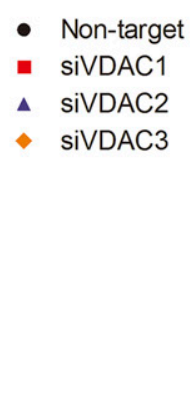

E

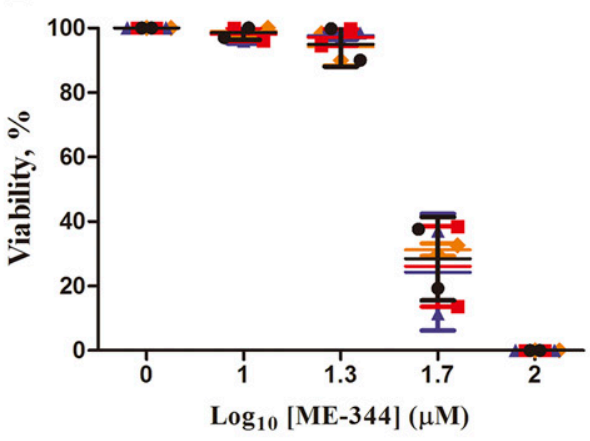

- Non-target

- siVDAC1

- siVDAC2

- siVDAC3

G

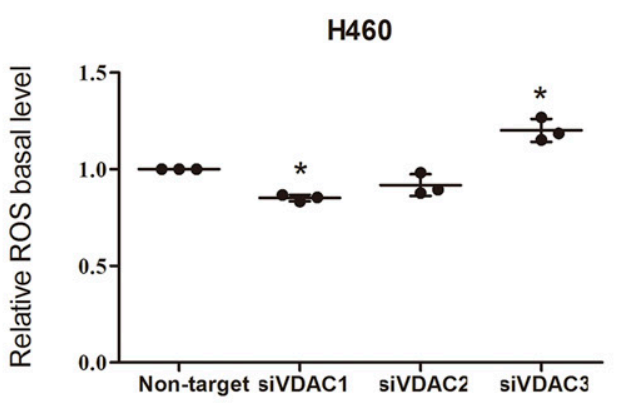

I

H596

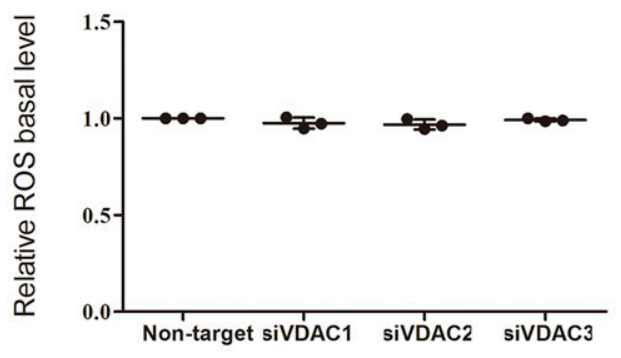

Fig. 2. Manipulation of VDAC1/2 expression impacts drug response. (A) Expression of VDAC isoforms VDAC1, VDAC2, and VDAC3 in H460 and H596 cell lines were determined by qPCR. (B and C) After transfection, mRNA and protein expression of the corresponding VDAC isoforms were assessed by qPCR and Western blot. (D and E) After transfection, H460 and H596 cell lines were treated with ME-344 (0-100 $\mu$ M) for 24 hours, and viabilities were determined by trypan blue assay in which \% viability was calculated relative to nontarget control. ( $\mathrm{F}$ and $\mathrm{H})$ Basal intracellular ROS levels determined with $\mathrm{CM}-\mathrm{H}_{2} \mathrm{DCFDA}$ by flow cytometry. ( $\mathrm{G}$ and I) Statistical analyses of cell ROS levels. Data are derived from three independent experiments and presented as mean \pm S.D. in the scatter plots. ${ }^{*} P<0.05$; vs. the untreated control by Student's $t$ test. 
A

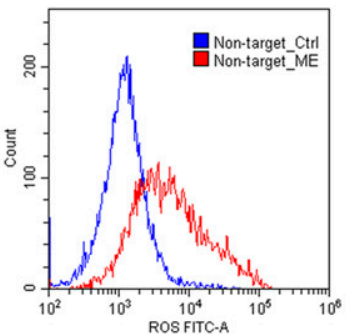

\section{E}

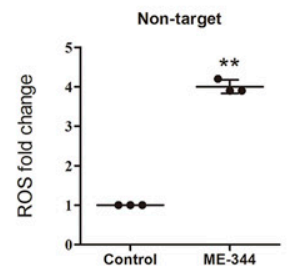

B

VDAC1 KD

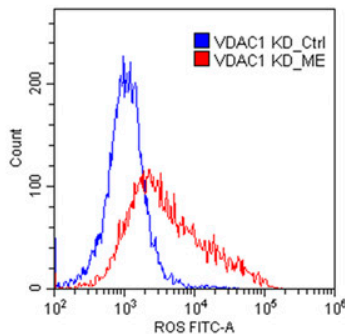

$\mathbf{F}$

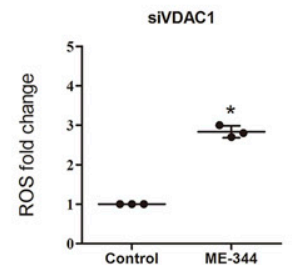

C $\quad$ DAC2 KD

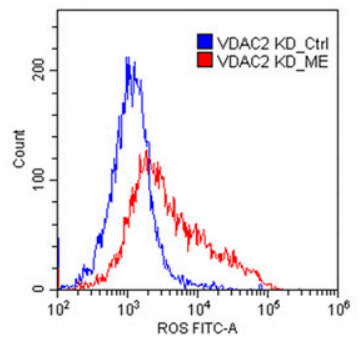

G

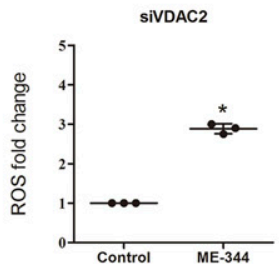

D

VDAC3 KD

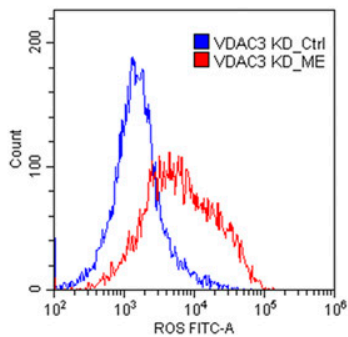

H

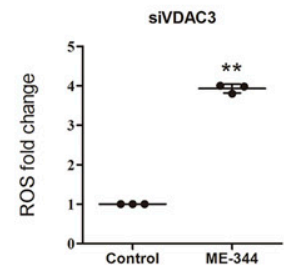

Fig. 3. Manipulation of VDAC1/2 expression impacts ROS production. (A-D) Cells were treated with their $\mathrm{IC}_{50} \mathrm{~S}$ of ME-344 for 24 hours, and intracellular ROS levels were determined with CM- $\mathrm{H}_{2}$ DCFDA by flow cytometry. $(\mathrm{E}-\mathrm{H})$ Statistical analyses of cell ROS levels. Data are derived from three independent experiments and presented as means \pm S.D. in the scatter plots. $* P<0.05 ; * * P<0.01$ vs. the untreated control by Student's $t$ test. Ctrl, control.

band, whereas the analysis described in this report was designed to discern differentially enriched proteins from full protein profiles. The relative abundance of proteins enriched by M2F-conjugated beads was compared with control resin measured by label-free proteomics (Supplemental Table 1). Twelve proteins were enriched by M2F beads, as determined by a $>2.5 \log _{2}$ fold change
A

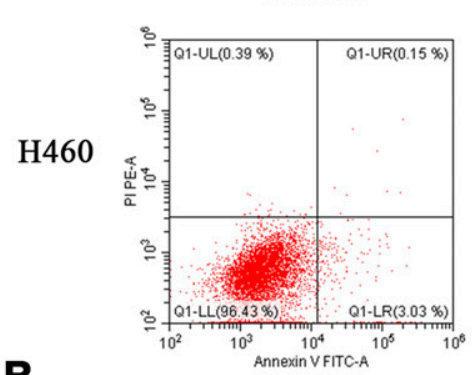

B

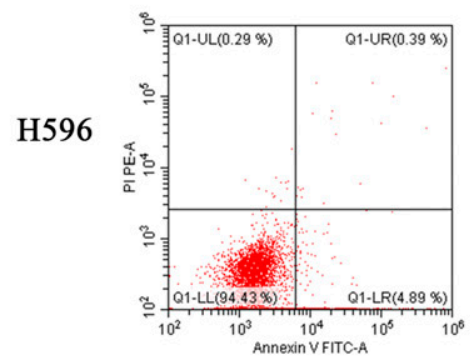

$7.5 \mu \mathrm{M}$
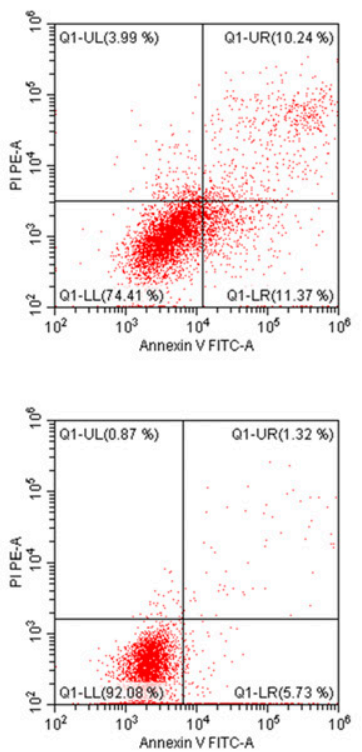

$15 \mu \mathrm{M}$
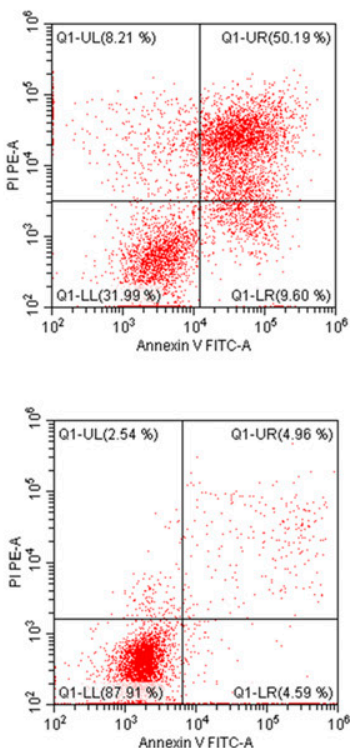

$30 \mu \mathrm{M}$
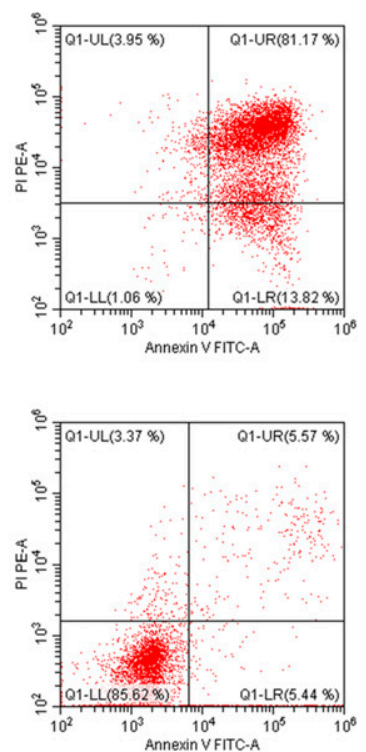

C
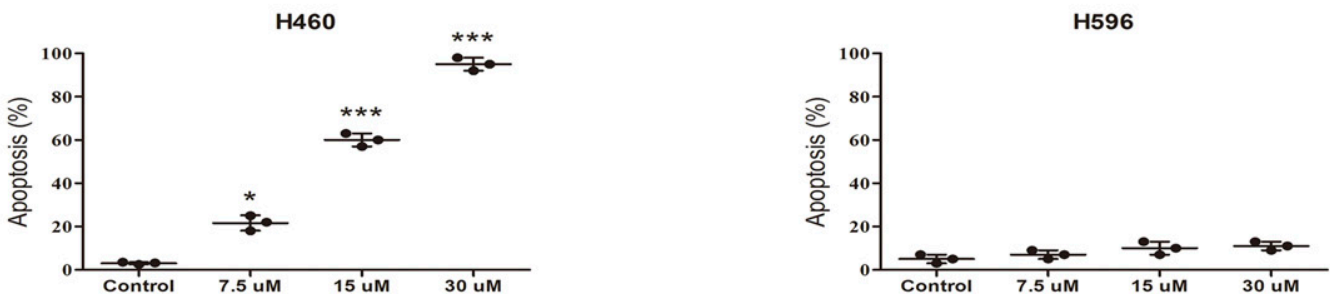

Fig. 4. Impact of ME-344 on apoptosis. (A and B) ME-344-treated cells were cultured for 24 hours prior to staining with Annexin V and PI. (C and D) Statistical analyses of cell apoptosis. Live: Annexin V and PI double negative cells. Apoptosis: Annexin V positive, PI positive, or negative cells. Data are derived from three independent experiments and presented as mean \pm S.D. in the scatter plots. ${ }^{*} P<0.05$; ${ }^{* * *} P<0.001$ vs. the untreated control by Student's $t$ test.PI, Propidium iodide. 
A

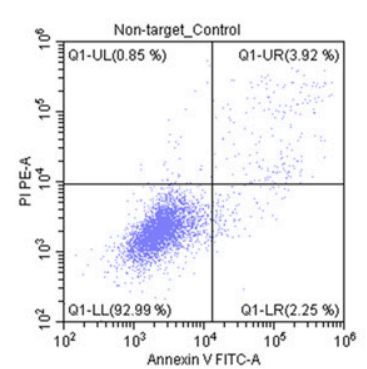

B

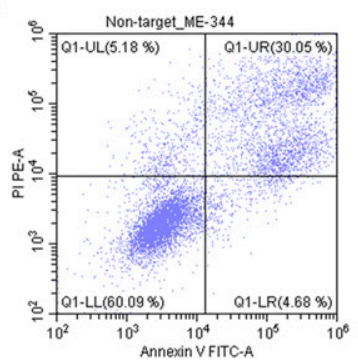

I

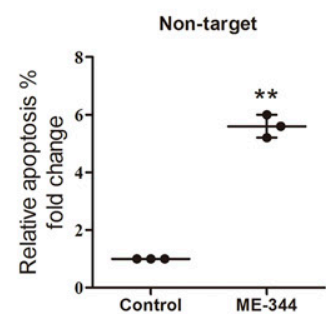

C

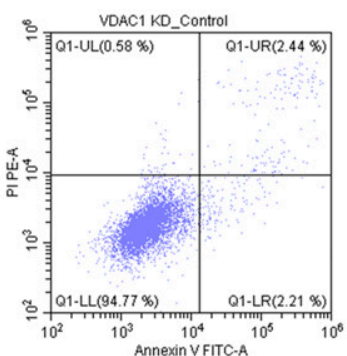

D

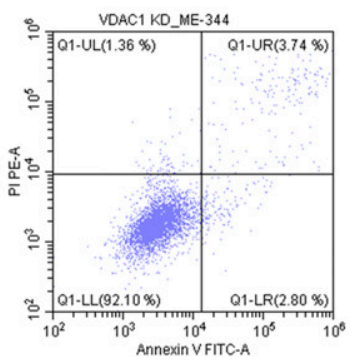

J

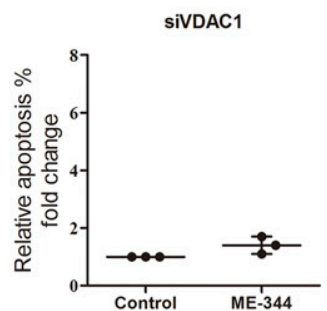

E

VDAC2 KD

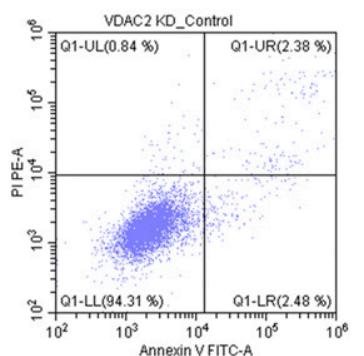

F

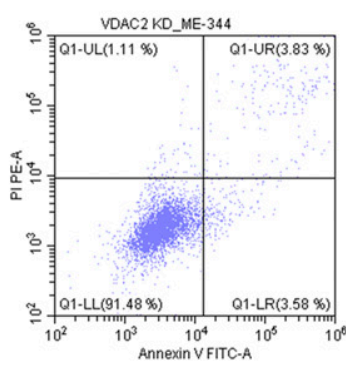

K

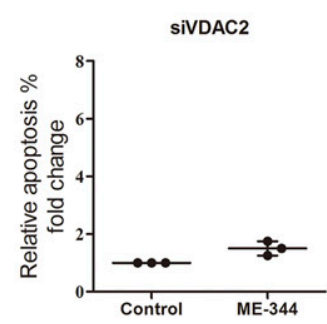

G
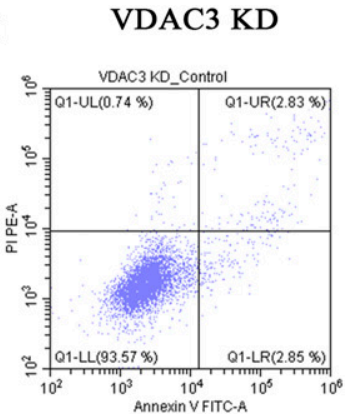

H

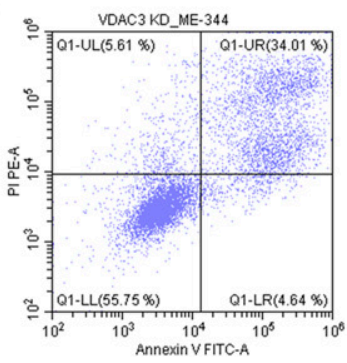

L

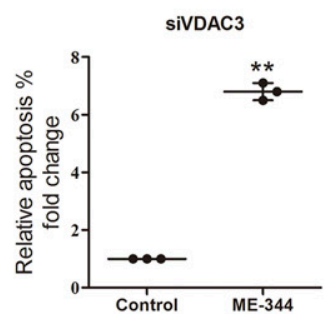

Fig. 5. Manipulation of VDAC1/2 expression impacts apoptosis. $(\mathrm{A}-\mathrm{H}) \mathrm{ME}-344$-treated (at their $\mathrm{IC}_{50} \mathrm{~s}: 10.05 \pm 0.28,14.74 \pm 0.32,13.81 \pm 0.23,9.38 \pm$ $0.29 \mu \mathrm{M}$, respectively) VDAC knockdown cells were cultured for 24 hours prior to staining with Annexin V and PI. (I-L) Statistical analyses of cell apoptosis. Live: Annexin V and PI double negative cells. Apoptosis: Annexin V positive, PI positive, or negative cells. Data are derived from three independent experiments and presented as mean \pm S.D. in the scatter plots. $* * P<0.01$ vs. the untreated control by Student's $t$ test. KD, Knock down; PI, Propidium iodide.

compared with control beads. These included VDAC1 and 2, enriched by $3.55-$ and $3.25-\log _{2}$-fold, respectively (Fig. 1A). Enriched proteins were queried for known interactions, and the resulting protein interaction networks were uploaded and merged with the drug-protein interaction network in Cytoscape. This analysis revealed VDAC1 and 2 were central to the network, interacting with five of the other enriched proteins, indicating direct binding of the drug to VDAC and enrichment of VDAC-binding proteins (Fig. 1B). Functional annotations associated with VDAC1 and 2 are shown in Fig. 1C.

VDAC Expression, ROS Production, and Drug Response. As assessed by qPCR, all three VDAC isoforms were expressed in both H460 and H596 cells. Proportionally, VDAC2 (48\% and 60\%) was the most abundant, followed by VDAC1 (30\% and 27\%) and VDAC3 (22\% and 13\%, respectively; Fig. 2A). We previously established that $\mathrm{H} 460$ cells are sensitive and H596 cells are intrinsically resistant to ME-344 and found no significant difference of basal protein levels of VDAC1 and VDAC2 in H460 and H596 cells. However, when challenged with ME-344, fold changes of VDAC1 and VDAC2 were both more pronounced in H460 cells than H596 cells. Interestingly, Medical Research Council cell strain 5 cells, normal lung fibroblast cells, were more closely aligned to the resistant cells than to the sensitive cells in the expression of VDAC1 and VDAC2 after ME-344 treatment (Supplemental
Fig. 2) (Zhang et al., 2019b). Isoform-specific siRNAs selectively inhibited expression of VDAC1, 2, and 3, respectively. siRNA treatment decreased both mRNA and protein expression by approximately $90 \%$ for each isoform without causing compensatory increases on expression of the other isoforms (Fig. 2, B and C). Response to ME-344 (trypan blue assay) and ROS production were determined after individual knockdown of each isoform in comparison with non-target-treated cells. The cell viabilities were shown in Fig. 2, D and $\mathrm{E}$ and Supplemental Table 2. The $\mathrm{IC}_{50} \mathrm{~s}$ for ME-344 in siVDAC1 and siVDAC2 knockdown $\mathrm{H} 460$ cells increased by $46.7 \%$ and $37.4 \%$, respectively, compared with nontarget siRNAs. There were no significant changes of $\mathrm{IC}_{50} \mathrm{~s}$ in $\mathrm{H} 596$ cells. Knockdown of VDAC1 or 2 slightly decreased basal ROS levels, whereas VDAC 3 knockdown increased basal ROS levels in H460 cells (Fig. 2, F and G). ROS production of H596 cells was unaffected (Fig. 2, H and I). Since ROS can induce mitochondrial permeability transition pore opening, we measured how ROS levels were influenced by VDAC knockdown. Reduced VDAC1 or 2 levels resulted in decreased ROS generation, whereas knockdown of VDAC3 increased ROS production induced by ME-344 compared with nontarget siRNA (Fig. 3).

Knockdown of VDAC1 or 2 decreased apoptosis induced by ME-344 in H460 cells. The respective contributions of early- (Annexin V-FITC positive, PI negative) and late-stage 


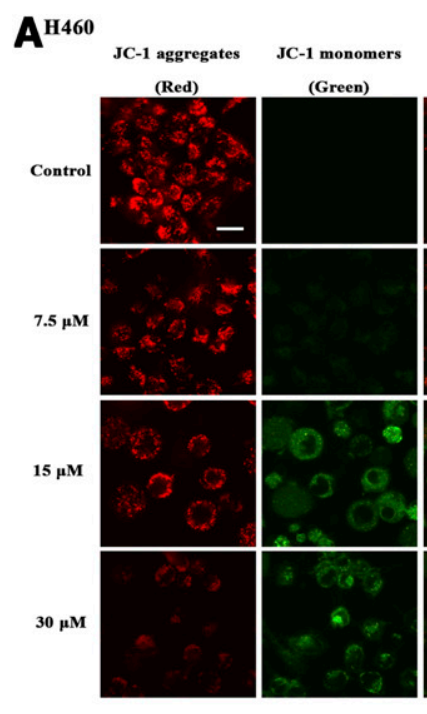

B

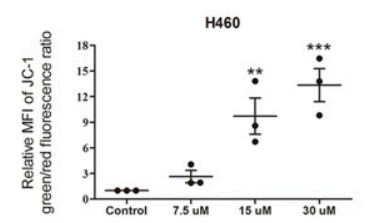

C

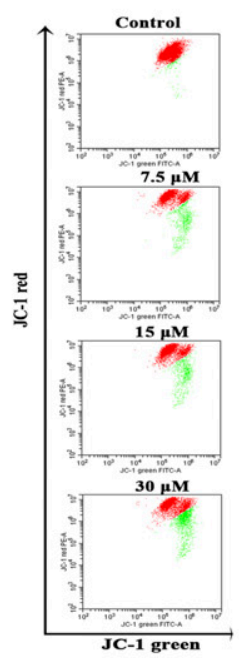

D

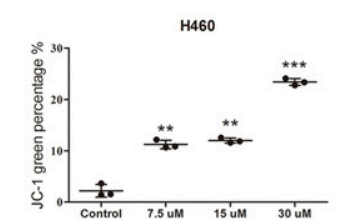

E ${ }^{H 596}$

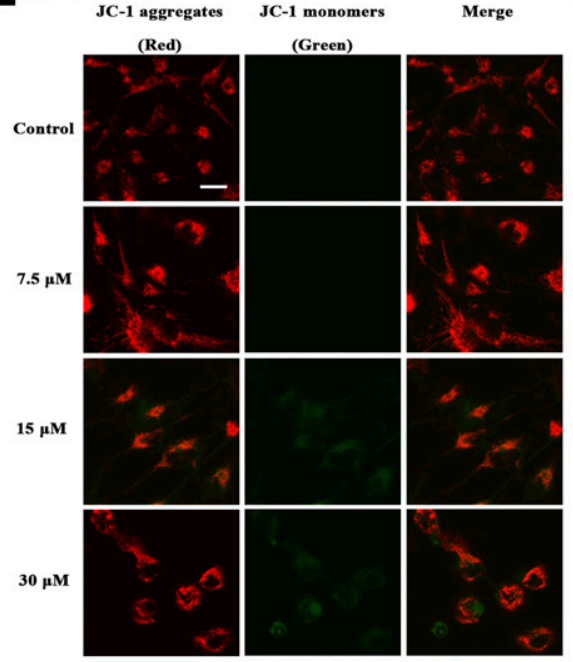

H

$\mathbf{F}$
G
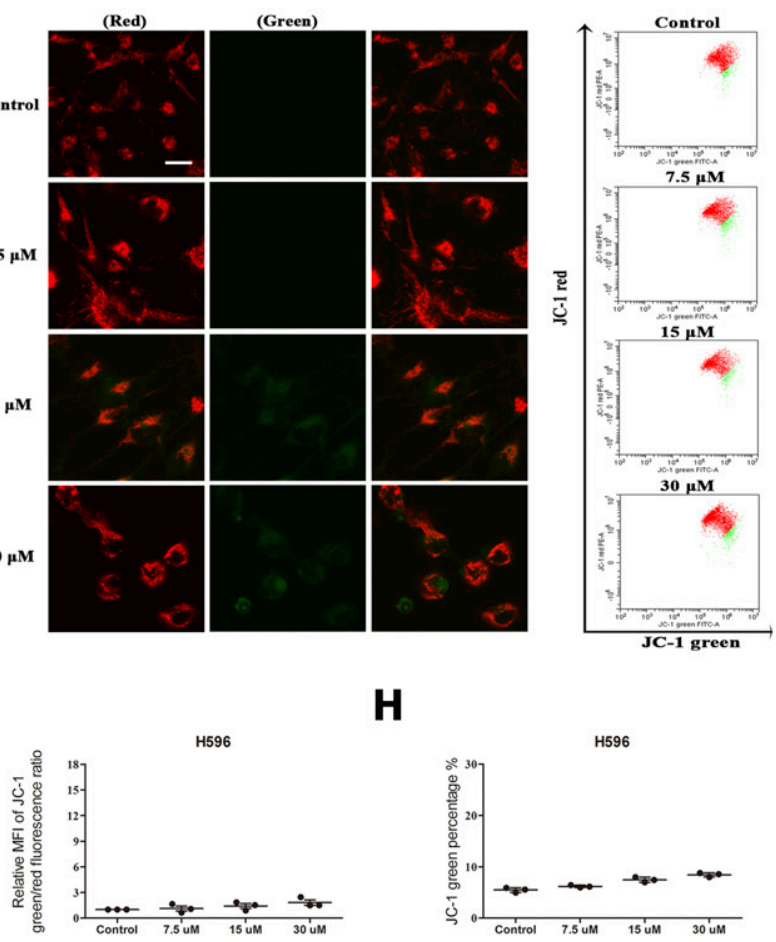

apoptosis (both Annexin V-FITC and PI positive) were measured in H460 cells after ME-344. The results showed that apoptosis rates increased in a dose-dependent manner in comparison with untreated cells. H596 cells were unaffected by similar drug concentrations (Fig. 4). Knockdown of either VDAC1 or 2 did, however, decrease the levels of apoptosis induced by ME-344 compared with nontarget siRNA (Fig. 5).

ME-344 Effects on Mitochondrial Membrane Potential. Loss of $\Delta \Psi_{\mathrm{m}}$ is an indicator of mitochondrial dysfunction that, in some cases, triggers the release of cytochrome $\mathrm{c}$ from mitochondria to the cytoplasm and activates the apoptotic caspase cascade. Previously, we showed that ME-344-induced apoptosis by activation of caspase-3 led to the cleavage of poly(ADP-ribose) polymerase (Zhang et al., 2019a). In this regard, ME-344 treatment decreased $\Delta \Psi_{\mathrm{m}}$ in a dose-dependent manner in $\mathrm{H} 460$ cells, as shown by confocal imaging of JC-1 fluorescence (Fig. 6, A and B) and flow cytometry (Fig. 6, C and D). These effects were not apparent for the resistant H596 cells (Fig. 6, E-H).

To assess the time at which $\Delta \Psi_{\mathrm{m}}$ began to change in $\mathrm{H} 460$ and H596 cells, we loaded cells with the potential-indicating fluorophore TMRM and subsequently imaged with confocal microscopy. Vehicle- or ME-344-treated cells in the same fields were followed for 3 hours. ME-344 induced significant depolarization of $\Delta \Psi_{\mathrm{m}}$ within 15 minutes in $\mathrm{H} 460$ cells (approximately 40\%) and by more than $60 \%$ at the end of the 3-hour treatment (Fig. 7, A and B). No significant depolarization was induced by ME-344 in H596 cells (Fig. 7, C and D).
These data were confirmed using flow cytometry (Fig. 7, E-H). To assess the impact of silencing VDAC on $\Delta \Psi_{\mathrm{m}}$, both H460 and H596 cells were transfected with either nontarget or VDAC1, 2, and 3 siRNAs and subsequently loaded with TMRM and imaged by confocal microscopy. In nontarget siRNA H460 cells, mitochondria expressed strong labeling with TMRM (Fig. 8A). Knockdown of VDAC isoforms 1, 2, and 3 decreased mitochondrial TMRM fluorescence by $19 \%, 18 \%$, and $26 \%$, respectively. The most significant decrease of $\Delta \Psi_{\mathrm{m}}$ occurred after knockdown of VDAC3, the least abundant isoform (Fig. 8B). ME-344 reduced the TMRM fluorescence in mitochondria, which was indicated by reduction in $\Delta \Psi_{\mathrm{m}}$ in H460 cells (Fig. 8, A and C). This drug-induced dissipation of $\Delta \Psi \mathrm{m}$ was diminished by knockdown of either VDAC1 or 2 in H460 cells (Fig. 8, A and C).

VDAC1 and 2 Are Required for Bax to Mediate ME-344-Induced Apoptosis in $\mathbf{H 4 6 0}$ Cells. Bax translocation from cytosol to mitochondria induces cytochrome c release, followed by caspase activation of apoptosis (Tang et al., 2006; George et al., 2010; Wang et al., 2013). To determine whether VDACs influence this process, Bax and cytochrome c levels were determined in mitochondria and cytosol in both H460 and H596 cells. ME-344 treatments of H460 with VDAC1 or 2 knockdown decreased both Bax translocation from cytosol to mitochondria and release of cytochrome c. These effects were not found in either VDAC3 or nontarget knockdowns (Fig. 9, A and B). Bax translocation and cytochrome c release were not affected in transfected 
A

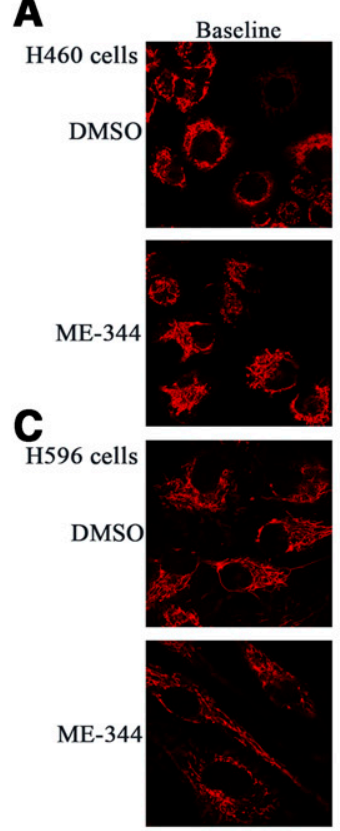

E

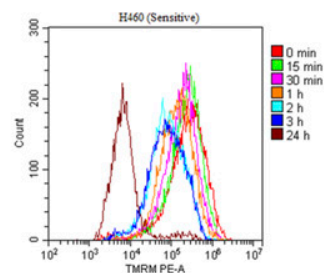

$15 \mathrm{~min}$
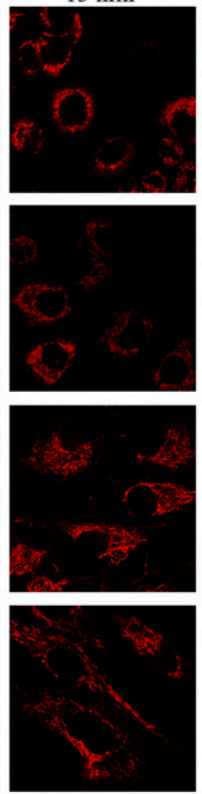

$\mathbf{F}$
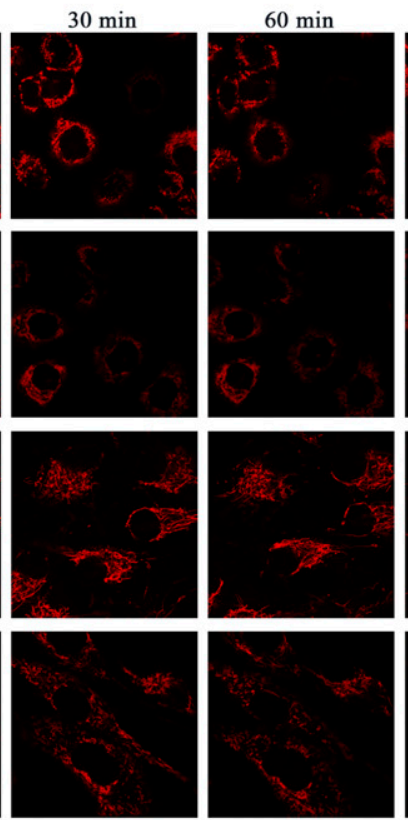

G

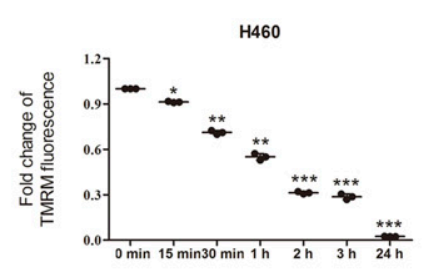

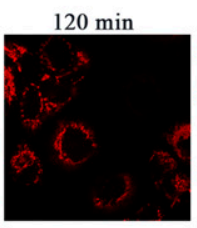
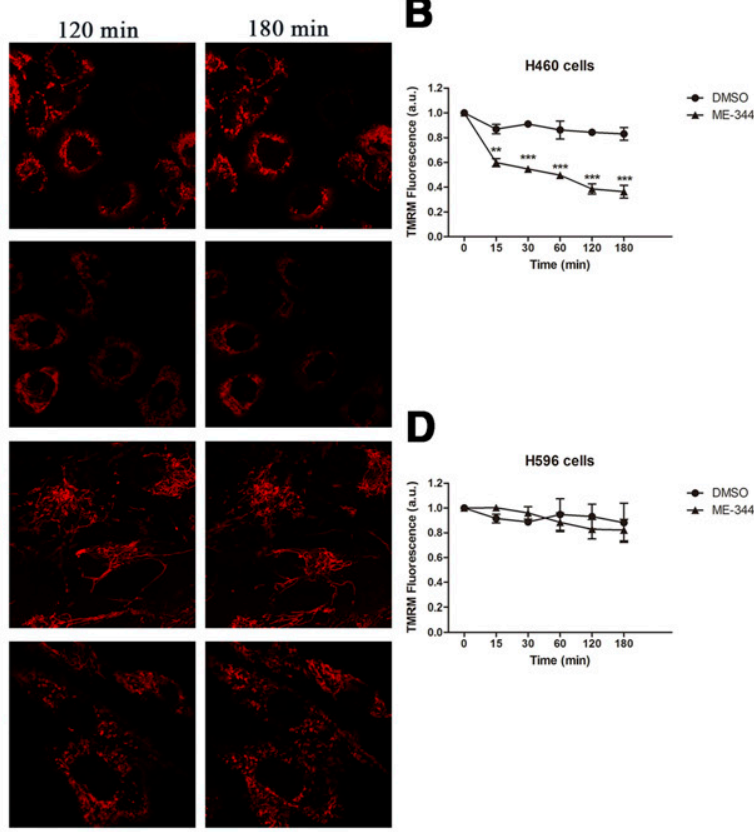

H

B
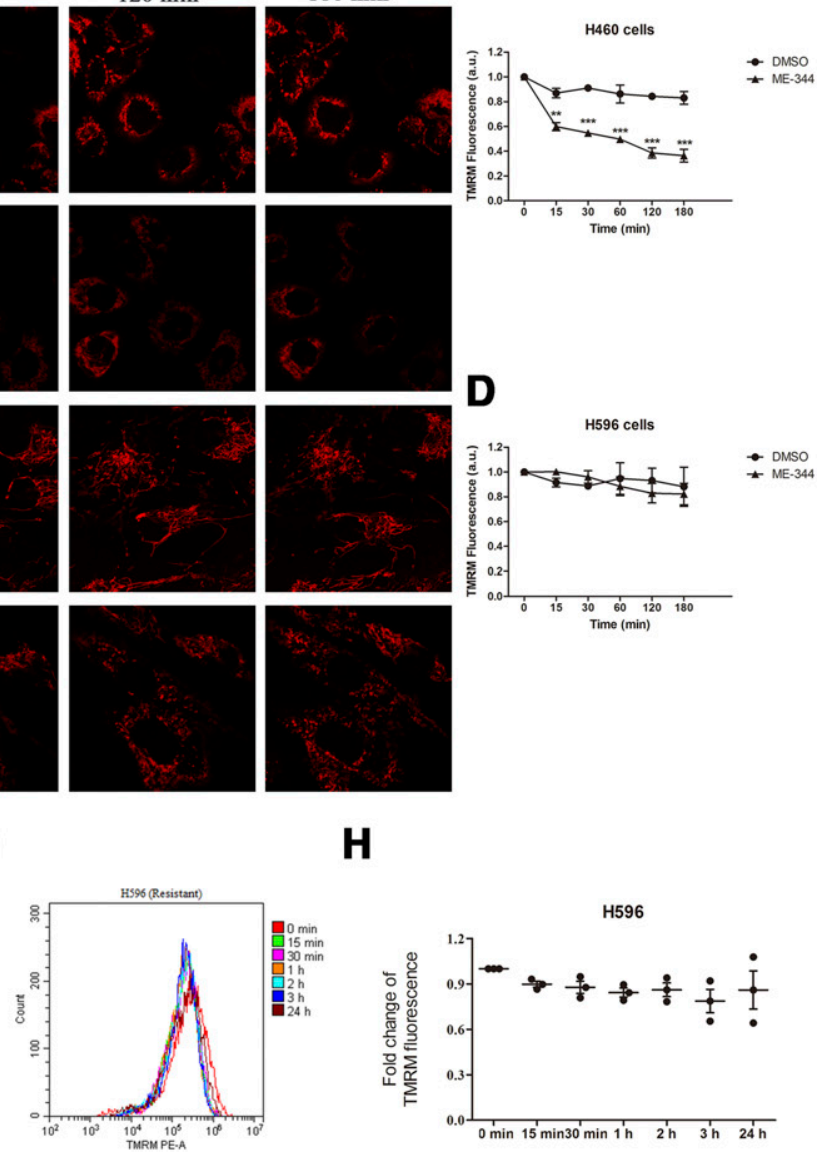

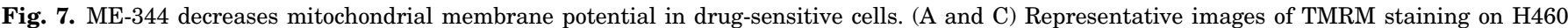

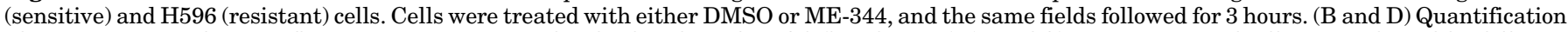

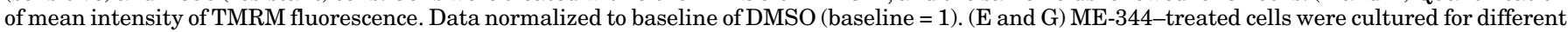

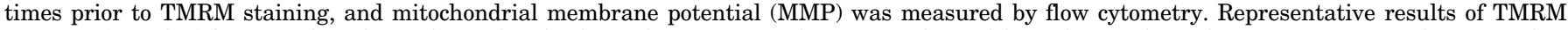

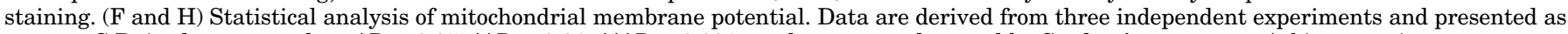
mean \pm S.D. in the scatter plots. $* P<0.05$; $* * P<0.01$; $* * P<0.001$ vs. the untreated control by Student's $t$ test. a.u., Arbitrary units.

H596 cells (Fig. 9, C and D). Taken together, these results showed that VDAC1 and 2, but not 3, influenced ME-344-mediated Bax translocation -mediated apoptosis in H460 cells.

\section{Discussion}

ME-344 has progressed into Phase I/II clinical trials (Bendell et al., 2015; Quintela-Fandino et al., 2018). An overview of preclinical studies indicates that the drug exerts its cytotoxic effects by targeting mitochondria in a number of different cancer types, including lung cancers (Zhang et al., 2019c). The observation that ME-344 can inhibit respiratory complexes with subsequent dysregulation of cellular ROS levels provided impetus for us to consider HO-1 as a potential drug target (Alvero et al., 2011; Lim et al., 2015; Manevich et al., 2016; Navarro et al., 2016). We used click chemistry approaches to confirm that HO-1 is a direct target for ME-344 binding and found that it time-dependently inhibited activity of $\mathrm{HO}-1$, bound to and altered HO-1 protein structure, and induced HO-1 translocation from ER to mitochondria. Its inhibition disrupted redox homeostasis leading to mitochodrial-mediated apoptosis (Zhang et al., 2019b). Our proteomic analysis of the click chemistry results also identified other mitochondrial proteins that were plausibly affected by ME-344 treatments. Among these, VDAC1 and VDAC2 were implicated (Zhang et al., $2019 \mathrm{~b})$. In light of the role of VDACs in response to oxidative stress (Noskov et al., 2016), this observation provided a platform for the present study, which was designed to interrogate whether drug effects on these mitochondrial membrane proteins were associated with drug cytotoxicity.

The present results showed that VDAC1 and 2 were enriched by 3.55 - and $3.25-\log _{2}$ fold-transformed intensities of drug binding when compared with their respective controls (Fig. 1). By using lung cancer cells that were either either intrinsically sensitive (H460) or resistant (H596) to the drug and employing siRNA to deplete each individual VDAC isoform, we were able to consider their relevance to drug effects. Our results indicated that genetic manipulation of either VDAC1 or 2 expression did influence drug sensitivity, intracellular ROS levels, and induction of apoptosis. Knockdown of either VDAC1 or 2 reduced intracellular ROS levels and made H460 cells less susceptible to ME-344 cytotoxicity. These observations are consistent with other reports showing that VDAC1 silencing lowered both ROS and cell death induced by either selenite (Tomasello et al., 2009) or cisplatin (Tajeddine et al., 2008). Contextually, ROSs are known to initiate mitochondrial permeability transition pore (mPTP) opening in mammalian cells (Zoratti and Szabo, 1994). As one 
A
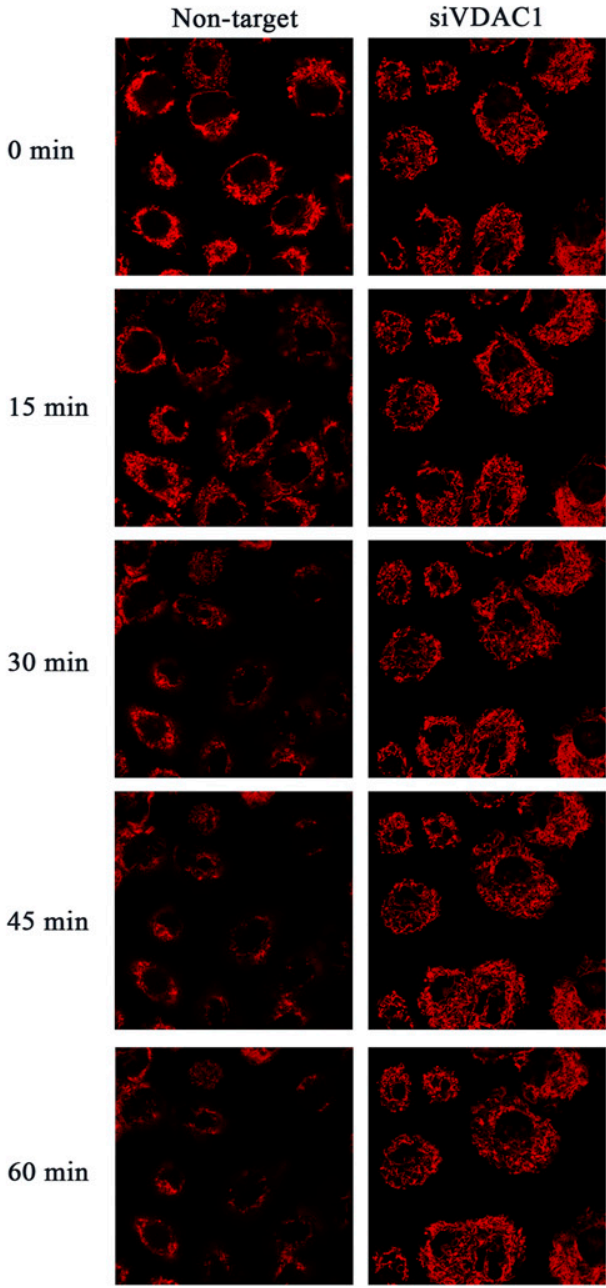

B

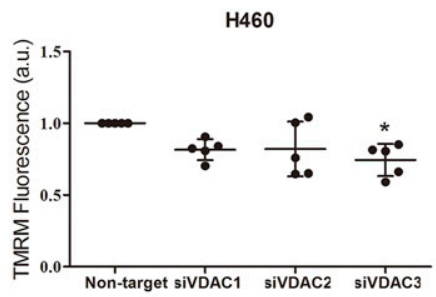

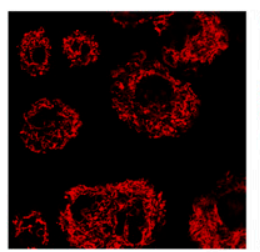

C
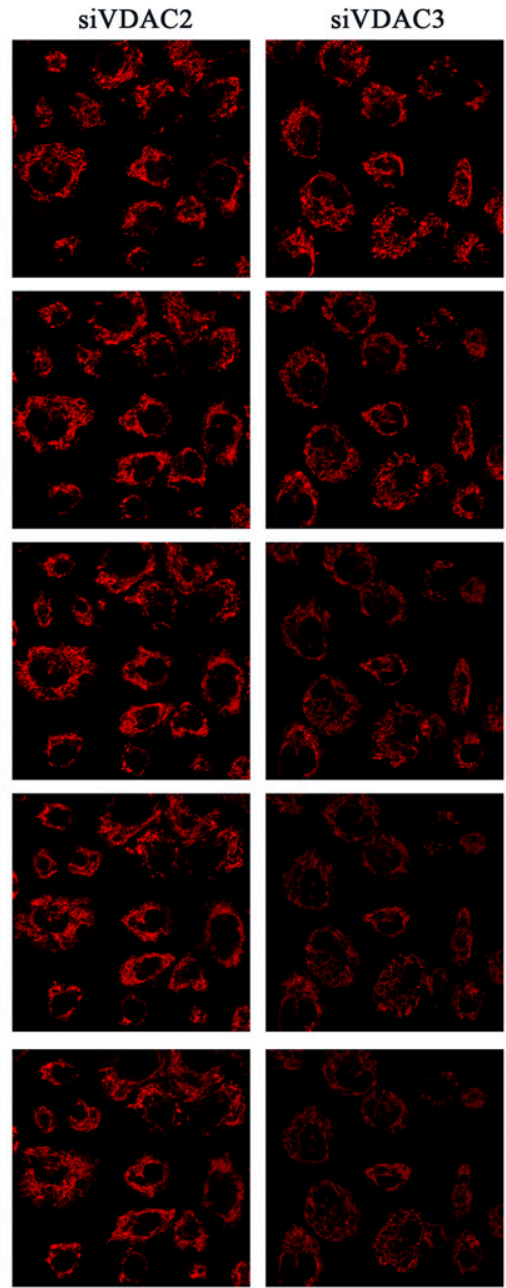

H460 cells

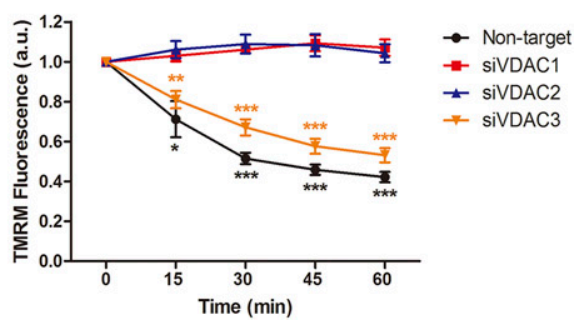

Fig, 8. VDAC knockdown decreases mitochondrial membrane potential, and VDAC1 and 2 knockdown induces recovery of mitochondrial membrane potential in sensitive cell line H460. (A) Representative images of TMRM staining on post-transfection H460 cells. Cells were treated with ME-344, and the same fields were followed for 1 hour after treatment. (B) Mean basal TMRM fluorescence after knockdown was plotted in comparison with transfection with nontarget siRNA. (C) Quantification is mean intensity of TMRM fluorescence. Data are normalized to baseline (baseline $=1$ ). Data are derived from three independent experiments and presented as mean \pm S.D. in the scatter plots. $* P<0.05 ; * * P<0.01 ; * * * P<$ 0.001 vs. the baseline control by Student's $t$ test. a.u., Arbitrary units. of the major putative components of mPTP, VDACs are localized in the outer mitochondrial membrane and are critical components to the formation of this pore (Vianello et al., 2012). Under physiologic conditions, VDACs facilitate metabolite exchange between mitochondria and cytosol, increase conductance linked to mPTP opening, and dissipate $\Delta \Psi_{\mathrm{m}}$ and eventual cytochrome c release, which are characteristics of the early stages of ME-344 toxicity leading to cell death (Shimizu et al., 1999; Tsujimoto and Shimizu, 2000). Our data show that ME-344 induced loss of $\Delta \Psi_{\mathrm{m}}$ in both nontarget and siVDAC3 H460 cells, but no $\Delta \Psi_{\mathrm{m}}$ changes were detected in siVDAC1- or siVDAC2-treated H460 cells. VDAC1 and 2 knockdown not only prevented the decrease in $\Delta \Psi_{\mathrm{m}}$ after ME-344 but also prevented Bax translocation and cytochrome c release. Bax, as a proapoptotic member of the Bcl-2 family of proteins, is a critical contributor to mitochondrial-mediated apoptosis pathways. Under nonstress conditions, Bax locates predominantly in the cytosol, but various exogenous stresses can cause its translocation to mitochondria as a prelude to apoptosis (Haneef et al., 2012; Li et al., 2013; Wang et al., 2013). Depletion of VDAC1 and 2 prevented both ME344-induced Bax translocation from cytosol to mitochondria and cytochrome c release from mitochondria to cytosol. These results indicate that in $\mathrm{H} 460$ cells the apoptosis caused by ME344 is contingent upon VDAC1 and 2, and disabling them can contribute to enhanced ROS, thus contributing to the cytoxic properties of ME-344.

There are other drugs that bind to VDAC2 and VDAC3. Erastin causes oxidative stress and mitochondrial dysfunction (Yagoda et al., 2007) through iron accumulation, ROS production resulting in ferroptosis (Dixon et al., 2012), which is reversible by iron chelation or antioxidants (Xu et al., 2019). 


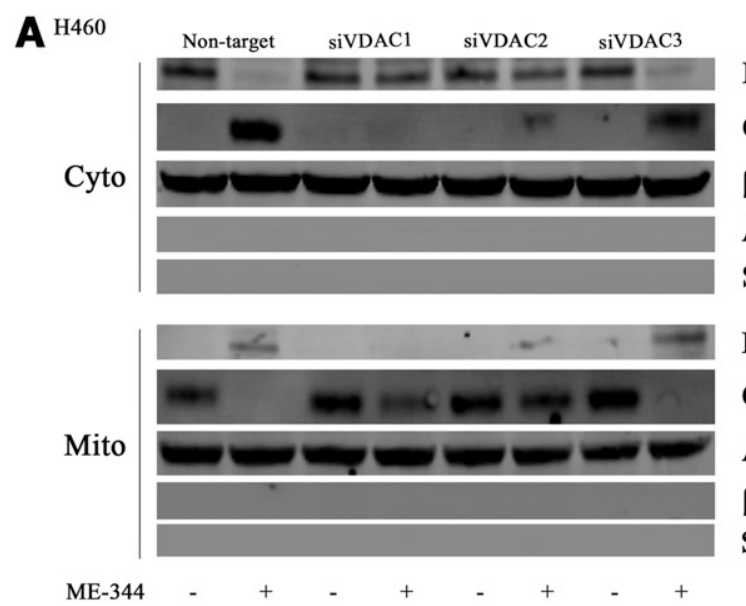

Bax
Cyt c
$\beta$-actin
ATP5A
SERCA
Bax
Cyt c
ATP5A
$\beta$-actin
SERCA


safety, and pharmacokinetics study of ME-344 as a single agent in patients with refractory solid tumors. Cancer 121:1056-1063.

Ben-Hail D, Begas-Shvartz R, Shalev M, Shteinfer-Kuzmine A, Gruzman A, Reina S, De Pinto V, and Shoshan-Barmatz V (2016) Novel compounds targeting the mitochondrial protein VDAC1 inhibit apoptosis and protect against mitochondrial dysfunction. J Biol Chem 291:24986-25003.

Brenner C and Grimm S (2006) The permeability transition pore complex in cancer cell death. Oncogene 25:4744-4756.

Caterino M, Ruoppolo M, Mandola A, Costanzo M, Orrù S, and Imperlini E (2017) Protein-protein interaction networks as a new perspective to evaluate distinct functional roles of voltage-dependent anion channel isoforms. Mol Biosyst 13:2466-2476.

Chin HS, Li MX, Tan IKL, Ninnis RL, Reljic B, Scicluna K, Dagley LF, Sandow JJ, Kelly GL, Samson AL, et al. (2018) VDAC2 enables BAX to mediate apoptosis and limit tumor development. Nat Commun 9:4976.

Colombini M (1989) Voltage gating in the mitochondrial channel, VDAC. J Membr Biol 111:103-111.

Dadsena S, Bockelmann S, Mina JGM, Hassan DG, Korneev S, Razzera G, Jahn H, Niekamp P, Müller D, Schneider M, et al. (2019a) Ceramides bind VDAC2 to trigger mitochondrial apoptosis. Nat Commun 10:1832.

Dadsena S, Hassan DG, and Holthuis JCM (2019b) Unraveling the molecular principles by which ceramides commit cells to death. Cell Stress 3:280-283.

Davis AP, Grondin CJ, Johnson RJ, Sciaky D, McMorran R, Wiegers J, Wiegers TC, and Mattingly CJ (2019) The comparative toxicogenomics database: update 2019 Nucleic Acids Res 47:D948-D954.

De Pinto V, Guarino F, Guarnera A, Messina A, Reina S, Tomasello FM, Palermo V, and Mazzoni C (2010) Characterization of human VDAC isoforms: a peculiar function for VDAC3? Biochim Biophys Acta 1797:1268-1275.

DeHart (2017) Erastin-Like Anti-Warburg Agents Prevent Mitochondrial Depolarization Induced by Free Tubulin and Decrease Lactate Formation in Cancer Cells. SLAS Discov.

Diamond JR, Goff B, Forster MD, Bendell JC, Britten CD, Gordon MS, Gabra H, Waterhouse DM, Poole M, Ross Camidge D, et al. (2017) Phase Ib study of the mitochondrial inhibitor ME-344 plus topotecan in patients with previously treated, locally advanced or metastatic small cell lung, ovarian and cervical cancers. Invest New Drugs 35:627-633.

Ding WX, Shen HM, and Ong CN (2001) Pivotal role of mitochondrial $\mathrm{Ca}(2+)$ in microcystin-induced mitochondrial permeability transition in rat hepatocytes. Biochem Biophys Res Commun 285:1155-1161.

Dixon SJ, Lemberg KM, Lamprecht MR, Skouta R, Zaitsev EM, Gleason CE, Patel DN, Bauer AJ, Cantley AM, Yang WS, et al. (2012) Ferroptosis: an iron-dependent form of nonapoptotic cell death. Cell 149:1060-1072.

Fang (2018) VDAC Regulation: A Mitochondrial Target to Stop Cell Proliferation. Adv Cancer Res.

George NM, Targy N, Evans JJ, Zhang L, and Luo X (2010) Bax contains two functional mitochondrial targeting sequences and translocates to mitochondria in a conformational change- and homo-oligomerization-driven process. J Biol Chem 285:1384-1392

Gincel D, Zaid H, and Shoshan-Barmatz V (2001) Calcium binding and translocation by the voltage-dependent anion channel: a possible regulatory mechanism in mitochondrial function. Biochem $J$ 358:147-155

Godbole A, Varghese J, Sarin A, and Mathew MK (2003) VDAC is a conserved element of death pathways in plant and animal systems. Biochim Biophys Acta 1642:87-96.

Haneef J, Parvathy M, Thankayyan R SK, Sithul H, and Sreeharshan S (2012) Bax translocation mediated mitochondrial apoptosis and caspase dependent photosensitizing effect of Ficus religiosa on cancer cells [published correction appears in PLoS One (2012) 7]. PLoS One 7:e40055.

Heslop (2020) JNK activation and translocation to mitochondria mediates mitochondrial dysfunction and cell death induced by VDAC opening and sorafenib in hepatocarcinoma cells. Biochem Pharmacol.

Hodge T and Colombini M (1997) Regulation of metabolite flux through voltagegating of VDAC channels. J Membr Biol 157:271-279.

Lemasters (2007) Imaging of mitochondrial polarization and depolarization with cationic fluorophores. Methods Cell Biol.

Li Z, Meng J, Xu TJ, Qin XY, and Zhou XD (2013) Sodium selenite induces apoptosis in colon cancer cells via Bax-dependent mitochondrial pathway. Eur Rev Med Pharmacol Sci 17:2166-2171.

Lim SC, Carey KT, and McKenzie M (2015) Anti-cancer analogues ME-143 and ME344 exert toxicity by directly inhibiting mitochondrial NADH: ubiquinone oxidoreductase (Complex I). Am J Cancer Res 5:689-701.

Lipper CH, Stofleth JT, Bai F, Sohn YS, Roy S, Mittler R, Nechushtai R, Onuchic JN, and Jennings PA (2019) Redox-dependent gating of VDAC by mitoNEET. Proc Natl Acad Sci USA 116:19924-19929.

Ma SB, Nguyen TN, Tan I, Ninnis R, Iyer S, Stroud DA, Menard M, Kluck RM, Ryan MT, and Dewson G (2014) Bax targets mitochondria by distinct mechanisms before or during apoptotic cell death: a requirement for VDAC2 or Bak for efficient Bax apoptotic function. Cell Death Differ 21:1925-1935.

Maldonado (2017) VDAC-Tubulin, an Anti-Warburg Pro-Oxidant Switch. Front Oncol PMC5256068.

Maldonado EN, Sheldon KL, DeHart DN, Patnaik J, Manevich Y, Townsend DM, Bezrukov SM, Rostovtseva TK, and Lemasters JJ (2013) Voltage-dependent anion channels modulate mitochondrial metabolism in cancer cells: regulation by free tubulin and erastin. $J$ Biol Chem 288:11920-11929.

Manevich Y, Reyes L, Britten CD, Townsend DM, and Tew KD (2016) Redox signaling and bioenergetics influence lung cancer cell line sensitivity to the isoflavone ME-344. J Pharmacol Exp Ther 358:199-208.

McCommis KS and Baines CP (2012) The role of VDAC in cell death: friend or foe? Biochim Biophys Acta 1818:1444-1450.

Navarro P, Bueno MJ, Zagorac I, Mondejar T, Sanchez J, Mourón S, Muñoz J, GómezLópez G, Jimenez-Renard V, Mulero F, et al. (2016) Targeting tumor mitochondrial metabolism overcomes resistance to antiangiogenics. Cell Rep 15:2705-2718.
Noskov SY, Rostovtseva TK, Chamberlin AC, Teijido O, Jiang W, and Bezrukov SM (2016) Current state of theoretical and experimental studies of the voltagedependent anion channel (VDAC). Biochim Biophys Acta 1858:1778-1790.

Okazaki M, Kurabayashi K, Asanuma M, Saito Y, Dodo K, and Sodeoka M (2015) VDAC3 gating is activated by suppression of disulfide-bond formation between the N-terminal region and the bottom of the pore. Biochim Biophys Acta 1848:3188-3196.

Otasek D, Morris JH, Boucas J, Pico AR, and Demchak B (2019) Cytoscape Automation: empowering workflow-based network analysis. Genome Biol 20:185.

Paddock ML, Wiley SE, Axelrod HL, Cohen AE, Roy M, Abresch EC, Capraro D, Murphy AN, Nechushtai R, Dixon JE, et al. (2007) MitoNEET is a uniquely folded $2 \mathrm{Fe} 2 \mathrm{~S}$ outer mitochondrial membrane protein stabilized by pioglitazone. Proc Natl Acad Sci USA 104:14342-14347.

Pillich RT, Chen J, Rynkov V, Welker D, and Pratt D (2017) NDEx: a community resource for sharing and publishing of biological networks. Methods Mol Biol 1558:271-301.

Pratt D, Chen J, Pillich R, Rynkov V, Gary A, Demchak B, and Ideker T (2017) NDEx 2.0: a clearinghouse for research on cancer pathways. Cancer Res 77:e58-e61.

Pratt D, Chen J, Welker D, Rivas R, Pillich R, Rynkov V, Ono K, Miello C, Hicks L, Szalma S, et al. (2015) NDEx, the network data exchange. Cell Syst 1:302-305.

Quintela-Fandino M, Apala JV, Salgado AC, Mouron SA, Guerra JA, Cortes MG, Morente M, and Manso L (2018) Abrogation of resistance against bevacizumab (Bev) by mitochondrial inhibition: a phase 0 randomized trial of Bev plus ME344 or placebo in early HER2-negative breast cancer (HERNEBC) (Abstract). J Clin Oncol 36:2552-2552.

Reina S, Guarino F, Magrì A, and De Pinto V (2016) VDAC3 as a potential marker of mitochondrial status is involved in cancer and pathology. Front Oncol 6:264.

Rostovtseva T and Colombini M (1996) ATP flux is controlled by a voltage-gated channel from the mitochondrial outer membrane. J Biol Chem 271:28006-28008. Schellenberg B, Wang P, Keeble JA, Rodriguez-Enriquez R, Walker S, Owens TW, Foster F, Tanianis-Hughes J, Brennan K, Streuli CH, et al. (2013) Bax exists in a dynamic equilibrium between the cytosol and mitochondria to control apoptotic priming. Mol Cell 49:959-971.

Shannon P, Markiel A, Ozier O, Baliga NS, Wang JT, Ramage D, Amin N, Schwikowski B, and Ideker T (2003) Cytoscape: a software environment for integrated models of biomolecular interaction networks. Genome Res 13:2498-2504.

Shimizu S, Narita M, and Tsujimoto Y (1999) Bcl-2 family proteins regulate the release of apoptogenic cytochrome $\mathrm{c}$ by the mitochondrial channel VDAC. Nature 399:483-487.

Suttner DM and Dennery PA (1999) Reversal of HO-1 related cytoprotection with increased expression is due to reactive iron. FASEB J 13:1800-1809.

Tajeddine N, Galluzzi L, Kepp O, Hangen E, Morselli E, Senovilla L, Araujo N, Pinna G, Larochette N, Zamzami N, et al. (2008) Hierarchical involvement of Bak, VDAC1 and Bax in cisplatin-induced cell death. Oncogene 27:4221-4232.

Tang HL, Le AH, and Lung HL (2006) The increase in mitochondrial association with actin precedes Bax translocation in apoptosis. Biochem $J$ 396:1-5.

Tomasello F, Messina A, Lartigue L, Schembri L, Medina C, Reina S, Thoraval D, Crouzet M, Ichas F, De Pinto V, et al. (2009) Outer membrane VDAC1 controls permeability transition of the inner mitochondrial membrane in cellulo during stress-induced apoptosis. Cell Res 19:1363-1376.

Tsujimoto Y and Shimizu S (2000) VDAC regulation by the Bcl-2 family of proteins. Cell Death Differ 7:1174-1181.

Vianello A, Casolo V, Petrussa E, Peresson C, Patui S, Bertolini A, Passamonti S, Braidot E, and Zancani M (2012) The mitochondrial permeability transition pore (PTP) - an example of multiple molecular exaptation? Biochim Biophys Acta 1817:2072-2086.

Wang Z, Lu W, Li Y, and Tang B (2013) Alpinetin promotes Bax translocation, induces apoptosis through the mitochondrial pathway and arrests human gastric cancer cells at the G2/M phase. Mol Med Rep 7:915-920.

Wettmarshausen J and Perocchi F (2017) Isolation of functional mitochondria from cultured cells and mouse tissues. Methods Mol Biol 1567:15-32.

Xu T, Ding W, Ji X, Ao X, Liu Y, Yu W, and Wang J (2019) Molecular mechanisms of ferroptosis and its role in cancer therapy. J Cell Mol Med 23:4900-4912.

Yagoda N, von Rechenberg M, Zaganjor E, Bauer AJ, Yang WS, Fridman DJ, Wolpaw AJ, Smukste I, Peltier JM, Boniface JJ, et al. (2007) RAS-RAF-MEK-dependent oxidative cell death involving voltage-dependent anion channels. Nature 447:864-868. Youle RJ and Strasser A (2008) The BCL-2 protein family: opposing activities that mediate cell death. Nat Rev Mol Cell Biol 9:47-59.

Yuan S, Fu Y, Wang X, Shi H, Huang Y, Song X, Li L, Song N, and Luo Y (2008) Voltage-dependent anion channel 1 is involved in endostatin-induced endothelial cell apoptosis. FASEB J 22:2809-2820.

Zaid H, Abu-Hamad S, Israelson A, Nathan I, and Shoshan-Barmatz V (2005) The voltage-dependent anion channel-1 modulates apoptotic cell death. Cell Death Differ 12:751-760

Zhang H, Zhou L, Davies KJA, and Forman HJ (2019a) Silencing Bach1 alters agingrelated changes in the expression of Nrf2-regulated genes in primary human bronchial epithelial cells. Arch Biochem Biophys 672:108074.

Zhang L, Zhang J, Ye Z, Manevich Y, Ball LE, Bethard JR, Jiang YL, Broome AM, Dalton $\mathrm{AC}$, Wang GY, et al. (2019b) Isoflavone ME-344 disrupts redox homeostasis and mitochondrial function by targeting heme oxygenase 1. Cancer Res 79:4072-4085.

Zhang L, Zhang J, Ye Z, Townsend DM, and Tew KD (2019c) Pharmacology of ME344, a novel cytotoxic isoflavone. Adv Cancer Res 142:187-207.

Zoratti M and Szabó I (1994) Electrophysiology of the inner mitochondrial membrane. J Bioenerg Biomembr 26:543-553.

Zoratti M, Szabò I, and De Marchi U (2005) Mitochondrial permeability transitions: how many doors to the house? Biochim Biophys Acta 1706:40-52.

Address correspondence to: Kenneth D. Tew, Department of Cell and Molecular Pharmacology and Experimental Therapeutics, Medical University of South Carolina, 70 President St., DD410, Charleston, SC 29425. E-mail: tewk@musc.edu 